\title{
Patterns of Sedentary Time and Quality of Life in Women With Fibromyalgia: Cross-Sectional Study From the al-Ándalus Project
}

Blanca Gavilán-Carrera ${ }^{1,2,3}$, BSc; Víctor Segura-Jiménez ${ }^{4,5}$, PhD; Pedro Acosta-Manzano ${ }^{1,2,3}$, BSc; Milkana Borges-Cosic $^{1,2,3}, \mathrm{PhD}$; Inmaculada C Álvarez-Gallardo ${ }^{1,3,4}, \mathrm{PhD}$; Manuel Delgado-Fernández ${ }^{1,2,3}, \mathrm{PhD}$

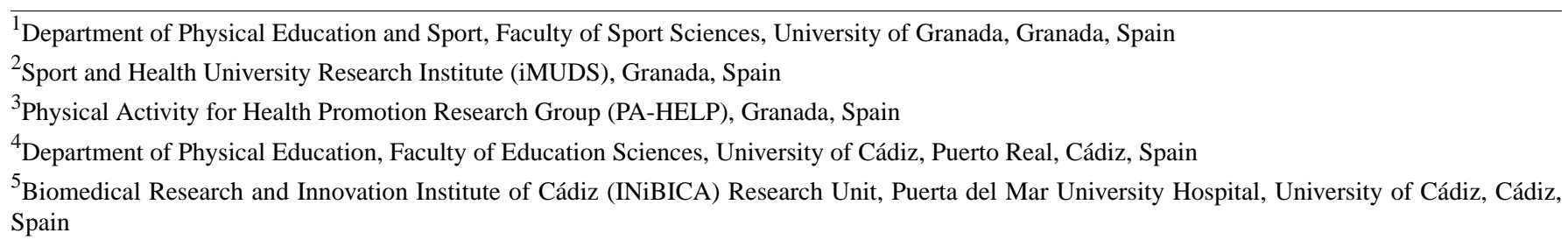

Corresponding Author:

Víctor Segura-Jiménez, $\mathrm{PhD}$

Department of Physical Education

Faculty of Education Sciences

University of Cádiz

Av República Saharaui s/n

11519

Puerto Real, Cádiz

Spain

Phone: 34956016219

Email: victor.segura@uca.es

\section{Abstract}

Background: Sedentary time (ST) has been associated with detrimental health outcomes in fibromyalgia. Previous evidence in the general population has shown that not only is the total amount of ST harmful but the pattern of accumulation of sedentary behaviors is also relevant to health, with prolonged unbroken periods (ie, bouts) being particularly harmful.

Objective: To examine the association of the patterns of ST with health-related quality of life (HRQoL) in women with fibromyalgia and to test whether these associations are independent of moderate-to-vigorous physical activity (MVPA).

Methods: A total of 407 women (mean 51.4 years of age [SD 7.6]) with fibromyalgia participated. ST and MVPA were measured with triaxial accelerometry. The percentage of ST accumulated in bouts and the frequency of sedentary bouts of different lengths ( $\geq 10 \mathrm{~min}, \geq 20 \mathrm{~min}, \geq 30 \mathrm{~min}$, and $\geq 60 \mathrm{~min}$ ) were obtained. Four groups combining total ST and sedentary bout duration ( $\geq 30$ min) were created. We assessed HRQoL using the 36-item Short-Form Health Survey (SF-36).

Results: A greater percentage of ST spent in all bout lengths was associated with worsened physical function, bodily pain, vitality, social function, and physical component summary (PCS) (all $P<.05$ ). In addition, a higher percentage of ST in bouts of 60 minutes or more was related to worsened physical role $(P=.04)$. A higher frequency of bouts was negatively associated with physical function, social function, the PCS ( $\geq 30 \mathrm{~min}$ and $\geq 60 \mathrm{~min}$ ), physical role ( $\geq 60 \mathrm{~min}$ ), bodily pain ( $\geq 60 \mathrm{~min}$ ), and vitality ( $\geq 20 \mathrm{~min}, \geq 30 \mathrm{~min}$, and $\geq 60 \mathrm{~min}$ ) (all $P<.05$ ). Overall, for different domains of HRQoL, these associations were independent of MVPA for higher bout lengths. Patients with high total ST and high sedentary bout duration had significantly worsened physical function (mean difference 8.73 units, 95\% CI 2.31-15.15; independent of MVPA), social function (mean difference 10.51 units, 95\% CI 2.59-18.44; not independent of MVPA), and PCS (mean difference 2.71 units, 95\% CI 0.36-5.06; not independent of MVPA) than those with low ST and low sedentary bout duration.

Conclusions: Greater ST in prolonged periods of any length and a higher frequency of ST bouts, especially in longer bout durations, are associated with worsened HRQoL in women with fibromyalgia. These associations were generally independent of MVPA.

(JMIR Mhealth Uhealth 2020;8(3):e14538) doi: 10.2196/14538 


\section{KEYWORDS}

GT3X+; accelerometry; sedentary behavior; symptomatology

\section{Introduction}

Fibromyalgia is a chronic and heterogeneous condition characterized by pain as the dominant symptom, which is frequently accompanied by fatigue, sleep disorders, or cognitive impairment [1]. Fibromyalgia patients, who tend to be highly sedentary, usually reduce their physical activity (PA) levels in order to avoid an aggravation of their symptomatology [2,3]. However, adopting this behavior might trigger a worsening of their condition [4-8]. Importantly, the risks of a sedentary lifestyle are present irrespective of the PA performed $[9,10]$. Considering that few patients with fibromyalgia fulfil the recommended level of moderate-to-vigorous PA (MVPA) [11], these patients are at an increased health risk not only for being highly sedentary but also for being inactive $[12,13]$. In the management of fibromyalgia, a graduated approach first focused on nonpharmacological modalities, and the improvement of health-related quality of life (HRQoL) is currently recommended [14]. Therefore, greater insights on how modifiable factors, such as daily sedentary time (ST) and PA, are related to HRQoL among these patients are warranted.

Emerging evidence in the general population has demonstrated that not only the total amount of ST but also the pattern of accumulation of sedentary behaviors is relevant to health [15-17]. Prolonged, unbroken periods (ie, bouts) of ST might be particularly harmful $[15,16]$ due to its relationship with detrimental effects on the metabolism [15-17]. In fibromyalgia, Ellingson et al demonstrated that both total ST, but especially sustained ST, can negatively influence pain modulation processes [18]. In addition, the frequency of sedentary bouts seems to be linked to health outcomes, with frequent interruptions in prolonged ST (ie, breaks) being beneficially related to markers of metabolic risk [19]. Although current PA recommendations emphasize the importance of reducing total ST [20], there is no information on how sedentary behavior patterns (ie, bout duration and frequency) should be modified to maximize health benefits. Sedentary patterns have been typically collected by accelerometers in the research field [17]. In contrast, mobile health (ie, mHealth) tools are more user-friendly devices that are widely used by consumers to track daily activity. These wearable devices, however, do not usually offer sedentary behavior information to users, although the inclusion of accelerometer sensors in wearable devices [21] would make it possible. Therefore, the analysis of the impact of different patterns of sustained ST on HRQoL in fibromyalgia could help in (1) the development of recommendations to reduce overall ST and in the interruption of potentially harmful bout lengths and (2) the implementation of future mHealth tools that deliver actual sedentary pattern information and potentially encourage this population to break prolonged ST.

Therefore, we aimed to examine (1) the association of the patterns of ST (ie, ST accumulated in bouts and frequency of sedentary bouts) in different bout lengths ( $\geq 10 \mathrm{~min}, \geq 20 \mathrm{~min}$, $\geq 30 \mathrm{~min}$, and $\geq 60 \mathrm{~min}$ ) with the HRQoL in women with fibromyalgia, (2) the combined association of total ST and sedentary bout duration with HRQoL, and (3) whether these associations are independent of MVPA.

\section{Methods}

\section{Recruitment}

A representative sample of fibromyalgia patients from the south of Spain-Andalusia-was recruited for the al-Ándalus project via fibromyalgia associations, internet advertisement, flyers, and email. Written informed consent from all participants $(\mathrm{N}=646)$ was obtained. Inclusion criteria for this study require that participants (1) be previously diagnosed by a rheumatologist and meet the 1990 American College of Rheumatology fibromyalgia criteria [1], (2) do not have either acute or terminal illness or severe cognitive impairment, and (3) are 65 years of age or younger. The flowchart of participants included in this study is shown in Figure 1. The Ethics Committee of the Hospital Virgen de las Nieves, Granada, Spain, reviewed and approved the study. 
Figure 1. Flow diagram of inclusion of women with fibromyalgia from the al-Ándalus project included in this study (N=407).

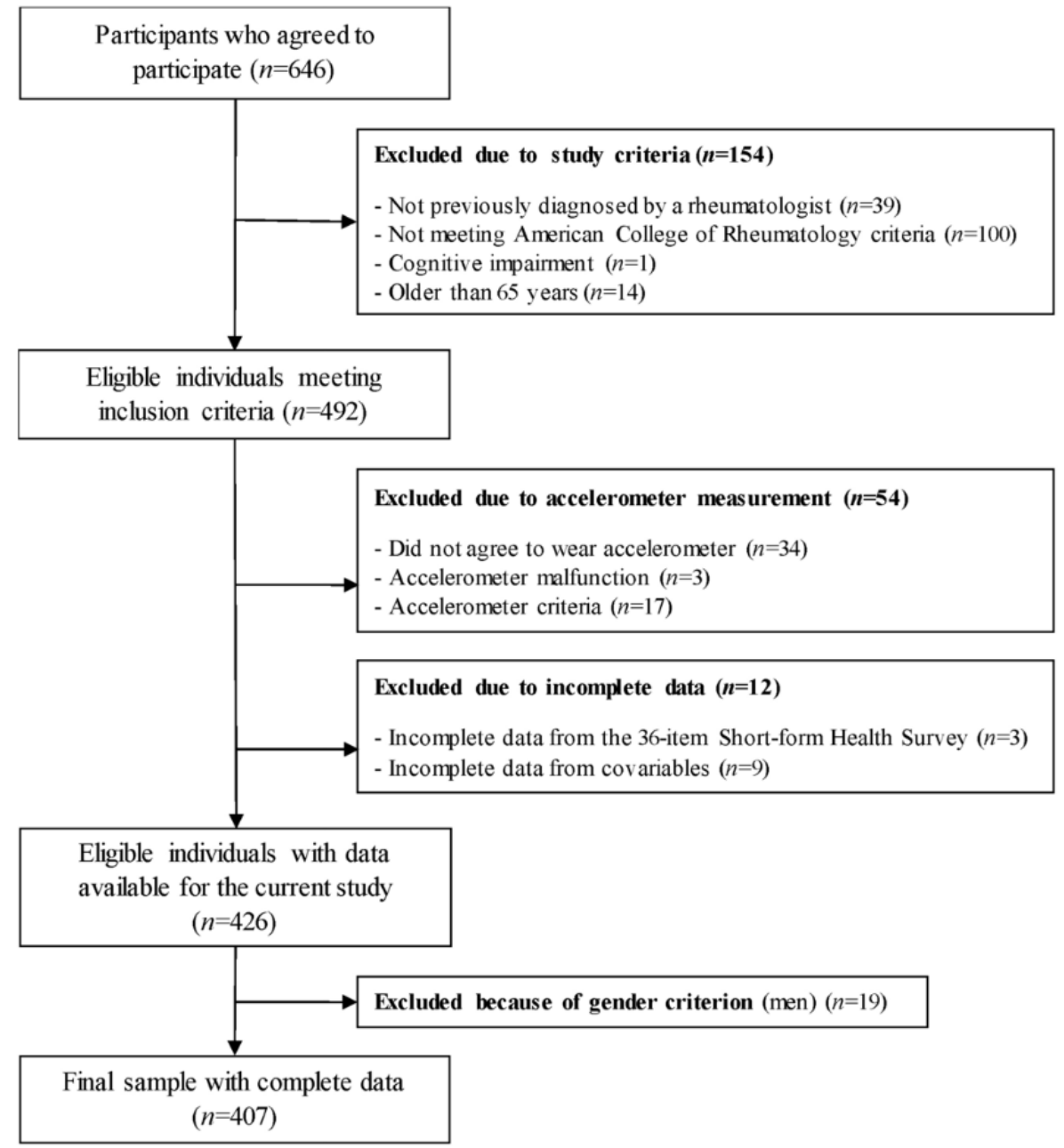

\section{Measurements}

\section{Sedentary Time and Physical Activity}

Patients wore the GT3X+ triaxial accelerometer (ActiGraph) on the hip for 9 days, 24 hours per day, except during water-based activities. Activity counts were measured at a rate of $30 \mathrm{~Hz}$ and stored at an epoch length of 1 minute [22]. Accelerometer-wearing time was obtained by subtracting the sleeping time and nonwear periods from each day. Sleeping time was obtained from a sleep diary, in which patients reported the time they went to bed and the time they woke up. Nonwear periods were obtained by applying Choi's algorithm [23]. Bouts of 90 continuous minutes of 0 counts were considered nonwear periods. To eliminate reactivity from the awareness of being monitored, we excluded PA data from the first day. The last day, when the device was returned, was excluded from the analysis as well. A total of 7 continuous days of recording, with a minimum of 10 valid hours per day, was the minimum criteria for being included in the study analysis.

ST and MVPA were calculated based upon recommended PA vector magnitude cut points [22,24]: 0-199 and $\geq 2690$ counts per minute (cpm), respectively. A sedentary bout was defined as the number of consecutive minutes during which the accelerometer registered less than $200 \mathrm{cpm}$. Four sedentary bout-length categories were used in this study: $\geq 10 \mathrm{~min}, \geq 20$ $\min , \geq 30 \mathrm{~min}$, and $\geq 60 \mathrm{~min}$. For each sedentary bout length, we obtained the following variables related to patterns of ST: (1) percentage of total ST accumulated in bouts (total time accumulated in bouts/total ST $\times 100)$ and (2) frequency of bouts (number of bouts/sedentary hours).

Data download, reduction, cleaning, and analyses were performed using ActiGraph's desktop software: ActiLife, version 6.11.7.

\section{Health-Related Quality of Life}

The HRQoL was assessed using the 36-item Short-Form Health Survey (SF-36) [25]. The SF-36 is composed of 36 items that assess eight dimensions of health (ie, physical functioning, physical role, bodily pain, general health, social functioning, emotional role, mental health, and vitality) and two component summary scores (ie, physical component summary [PCS] and mental component summary [MCS]). The score in each of the eight dimensions is standardized and ranges from 0 (worst health status) to 100 (best health status).

\section{Sociodemographic and Clinical Data}

We collected sociodemographic and clinical data using a self-reported questionnaire that included age, marital status (married/not married), education level (university/nonuniversity), and occupational status (working/not working). Patients also reported the consumption of 
antidepressants and analgesics (yes/no) during the previous 2 weeks.

\section{Anthropometry and Body Composition}

Weight $(\mathrm{kg})$ and total body fat $(\%)$ were measured using bioelectrical impedance with the InBody R20 (Biospace) body composition analyzer. Patients were asked neither to have a shower, practice intense PA, nor ingest large amounts of fluid and/or food in the 2 hours before the measurement. Patients removed their clothing and any metal objects from their bodies during the assessment.

\section{Impact of the Disease}

The Revised Fibromyalgia Impact Questionnaire (FIQR) [26] assesses overall fibromyalgia severity through a wide range of symptoms, comorbidities, and complaints related to this chronic condition. It is a self-administered questionnaire with 21 individual questions, with a rating scale of $0-10$. The adjusted FIQR total score ranges from 0 to 100 , with a higher score indicating greater impact of the syndrome on a person's life.

\section{Statistical Analysis}

Descriptive continuous data are shown as mean (SD), whereas categorical data are presented as n (\%). To test the association between patterns of ST and HRQoL, we used linear regression analysis. The eight dimensions and the two summary components of the SF-36 were introduced as dependent variables in the models in separate regression models. Patterns of ST (ie, percentage of ST accumulated in bouts and frequency of bouts in all bout lengths) were introduced individually as predictor variables. Two types of models were built: (1) model 1 was controlled for age, total body fat percentage, occupational status, medication for pain and depression, and accelerometer wear time and (2) model 2 included model 1 plus MVPA.

The combined association of total ST and prolonged sedentary bout duration with HRQoL was studied through analyses of covariance. The subject pool was divided into four groups according to the median value of total ST (3216 min/week) and the median value of sedentary bout durations of 30 continuous minutes or more $(47.7 \mathrm{~min})$. A minimum duration of 30 continuous minutes was used to define prolonged ST following the criteria of previous studies [27]. The four groups created were (1) low total ST ( $\leq$ the median value) + low sedentary bout duration ( $\leq$ the median value), (2) low total ST + high sedentary bout duration (> the median value), (3) high total ST (> the median value) + low sedentary bout duration, and (4) high total ST + high sedentary bout duration. The analyses were adjusted for age, total body fat percentage, occupational status, medication for pain and depression, and accelerometer-wear time. Additional analyses including MVPA as covariate were performed.

For analyses, we used IBM SPSS Statistics for Windows, version 20.0 (IBM Corp). The statistical significance was set at $P<.05$.

\section{Data Exclusion}

The final sample size included in the analyses comprised 407 women with fibromyalgia. The flow diagram of women with fibromyalgia included in this study is shown in Figure 1.

\section{Results}

Table 1 provides an overview of the patients' sociodemographic and clinical characteristics. Table 2 includes the information related to PA and ST pattern characteristics (ie, percentage of total ST and frequency of bouts) in different bout lengths.

The association of the percentage of ST accumulated in bouts of different lengths with the SF-36 domains are shown in Table 3. Greater percentages of ST spent in all bout lengths were associated with worse physical function, bodily pain, vitality, and social function domains and the PCS (beta from -.20 to -.10, all $P<.05)$. In addition, a higher percentage of ST spent in bouts of 60 minutes or more was related to a worsened physical role (beta=-.10, $P=.04$ ). Overall, these associations were independent of MVPA (all $P<.05$ ), except for the bodily pain (for bouts $\geq 10, \geq 20$, or $\geq 30 \mathrm{~min}$ ) and physical role domains. 
Table 1. Sociodemographic and clinical characteristics of the study participants $(\mathrm{N}=407)$.

\begin{tabular}{|c|c|}
\hline Variables & Mean $(\mathrm{SD})$ or $\mathrm{n}(\%)$ \\
\hline Age (years), mean (SD) & $51.4(7.6)$ \\
\hline Algometer score (18-144), mean (SD) & $43.2(13.4)$ \\
\hline Body mass index $\left(\mathrm{kg} / \mathrm{m}^{2}\right)$, mean $(\mathrm{SD})$ & $28.4(5.4)$ \\
\hline Total body fat (\%), mean (SD) & $40.1(7.6)$ \\
\hline FIQR $^{\mathrm{a}}$ score $(0-100)$, mean $(\mathrm{SD})$ & $64.4(16.7)$ \\
\hline \multicolumn{2}{|c|}{ Health-related quality of life, SF-36 ${ }^{b}$ score $(0-100)$, mean (SD) } \\
\hline Physical function & $39.2(18.9)$ \\
\hline Physical role & $33.2(21.2)$ \\
\hline Bodily pain & $21.2(14.7)$ \\
\hline General health & $28.5(15.3)$ \\
\hline Vitality & $22.3(17.7)$ \\
\hline Social functioning & $43.7(24.7)$ \\
\hline Emotional role & $56.9(27.9)$ \\
\hline Mental health & $46.2(19.7)$ \\
\hline Physical component & $29.5(6.9)$ \\
\hline Mental component & $36.0(11.6)$ \\
\hline \multicolumn{2}{|l|}{ Marital status, n (\%) } \\
\hline Married & $311(76.4)$ \\
\hline Not married & $96(23.6)$ \\
\hline \multicolumn{2}{|l|}{ Education level, $n$ (\%) } \\
\hline Nonuniversity & $349(85.7)$ \\
\hline University & $58(14.3)$ \\
\hline \multicolumn{2}{|l|}{ Current occupational status, n (\%) } \\
\hline Working & $107(26.3)$ \\
\hline Not working & $300(73.7)$ \\
\hline \multicolumn{2}{|l|}{ Drug consumption, n (\%) } \\
\hline Analgesics & $367(90.2)$ \\
\hline Antidepressants & $232(57.0)$ \\
\hline
\end{tabular}

${ }^{\mathrm{a}}$ FIQR: Revised Fibromyalgia Impact Questionnaire.

${ }^{\mathrm{b}}$ SF-36: 36-item Short-Form Health Survey. 
Table 2. Sedentary patterns and physical activity (PA) variables of the study participants ( $N=407)$.

\begin{tabular}{|c|c|}
\hline Sedentary behavior and PA & Mean (SD) \\
\hline Accelerometer-wear time & $923.0(78.9)$ \\
\hline \multicolumn{2}{|l|}{ Sedentary time (ST) } \\
\hline Minutes per day & $460.1(104.1)$ \\
\hline Percentage of wear time & $49.9(10.6)$ \\
\hline \multicolumn{2}{|l|}{ Light PA } \\
\hline Minutes per day & $418.6(91.8)$ \\
\hline Percentage of wear time & $45.3(9.1)$ \\
\hline \multicolumn{2}{|l|}{ Moderate PA } \\
\hline Minutes per day & $43.9(29.5)$ \\
\hline Percentage of wear time & $4.8(3.2)$ \\
\hline \multicolumn{2}{|l|}{ Vigorous PA } \\
\hline Minutes per day & $0.4(2.0)$ \\
\hline Percentage of wear time & $0.1(0.2)$ \\
\hline \multicolumn{2}{|l|}{ Moderate-to-vigorous PA } \\
\hline Minutes per day & $44.3(30.1)$ \\
\hline Percentage of wear time & $4.8(3.2)$ \\
\hline \multicolumn{2}{|l|}{ Patterns of ST of different bout lengths } \\
\hline \multicolumn{2}{|l|}{$\geq 10$-minute bout } \\
\hline Percentage of total ST accumulated (\%) & $59.2(11.2)$ \\
\hline Frequency of bouts (number of bouts/week) & $83.7(25.6)$ \\
\hline \multicolumn{2}{|l|}{$\geq 20$-minute bout } \\
\hline Percentage of total ST accumulated (\%) & $38.5(12.8)$ \\
\hline Frequency of bouts (number of bouts/week) & $34.3(14.6)$ \\
\hline \multicolumn{2}{|l|}{$\geq 30$-minute bout } \\
\hline Percentage of total ST accumulated (\%) & $26.7(12.3)$ \\
\hline Frequency of bouts (number of bouts/week) & $17.9(9.6)$ \\
\hline \multicolumn{2}{|l|}{$\geq 60$-minute bout } \\
\hline Percentage of total ST accumulated (\%) & $10.3(8.9)$ \\
\hline Frequency of bouts (number of bouts/week) & $4.3(3.7)$ \\
\hline
\end{tabular}


Table 3. Association of the percentage of sedentary time (ST) accumulated in bouts of different lengths with 36-item Short-Form Health Survey (SF-36) dimensions $(\mathrm{N}=407)$.

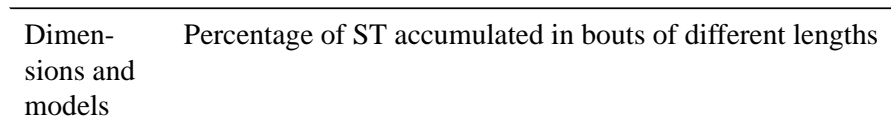

models

\begin{tabular}{|c|c|c|c|c|c|c|c|c|c|c|c|c|c|c|c|c|}
\hline & \multicolumn{4}{|c|}{$\geq 10$-minute bout } & \multicolumn{4}{|c|}{$\geq 20$-minute bout } & \multicolumn{4}{|c|}{$\geq 30$-minute bout } & \multicolumn{4}{|c|}{$\geq 60$-minute bout } \\
\hline & $\mathrm{B}^{\mathrm{a}}$ & SE & Beta $^{b}$ & $P$ & B & SE & Beta & $P$ & B & SE & Beta & $P$ & B & SE & Beta & $P$ \\
\hline \multicolumn{17}{|c|}{ Physical function } \\
\hline $\begin{array}{l}\text { Model } \\
1^{\mathrm{c}}\end{array}$ & -0.267 & 0.086 & -.159 & .002 & -0.253 & 0.074 & -.171 & .001 & -0.271 & 0.077 & -.176 & $<.001$ & -0.428 & 0.104 & -.201 & $<.001$ \\
\hline $\begin{array}{l}\text { Model } \\
2^{\mathrm{d}}\end{array}$ & -0.226 & 0.089 & -.134 & .01 & -0.221 & 0.077 & -.149 & .004 & -0.239 & 0.079 & -.156 & .002 & -0.396 & 0.105 & -.186 & $<.001$ \\
\hline
\end{tabular}

\section{Physical role}

$\begin{array}{lllllllllllllllll}\begin{array}{l}\text { Model } \\ 1\end{array} & -0.126 & 0.094 & -.067 & .18 & -0.137 & 0.081 & -.082 & .09 & -0.159 & 0.084 & -.092 & .06 & -0.239 & 0.114 & -.100 & .04 \\ \begin{array}{l}\text { Model } \\ 2\end{array} & -0.063 & 0.097 & -.033 & .52 & -0.089 & 0.083 & -.054 & .29 & -0.115 & 0.086 & -.067 & .18 & -0.195 & 0.115 & -.082 & .09\end{array}$

Bodily pain

\begin{tabular}{|c|c|c|c|c|c|c|c|c|c|c|c|c|c|c|c|c|}
\hline $\begin{array}{l}\text { Model } \\
1\end{array}$ & -0.130 & 0.065 & -.099 & .045 & -0.108 & 0.056 & -.094 & .05 & -0.114 & 0.058 & -.096 & .048 & -0.190 & 0.078 & -.115 & .02 \\
\hline $\begin{array}{l}\text { Model } \\
2\end{array}$ & -0.106 & 0.067 & -.081 & .12 & -0.089 & 0.058 & -.077 & .13 & -0.096 & 0.059 & -.080 & .11 & -0.171 & 0.079 & -.104 & .03 \\
\hline
\end{tabular}

\section{General health}

$\begin{array}{llllllllllllllllll}\text { Model } & -0.034 & 0.069 & -.025 & .62 & -0.048 & 0.060 & -.040 & .42 & -0.058 & 0.062 & -.047 & .35 & -0.076 & 0.084 & -.044 & .37 \\ 1 & & & & & & & & & & & & & & & \\ \begin{array}{l}\text { Model } \\ 2\end{array} & -0.029 & 0.072 & -.021 & .69 & -0.046 & 0.062 & -.038 & .46 & -0.056 & 0.064 & -.045 & .38 & -0.073 & 0.085 & -.042 & .39 \\ 2 & & & & & & & & & & & & & & & \end{array}$

\section{Vitality}

$\begin{array}{lllllllllllllllll}\text { Model } & -0.252 & 0.080 & -.160 & .002 & -0.204 & 0.069 & -.148 & .003 & -0.204 & 0.072 & -.142 & .004 & -0.278 & 0.097 & -.140 & .004 \\ 1 & & & & & & & & & & & & & & & \\ \begin{array}{l}\text { Model } \\ 2\end{array} & -0.209 & 0.083 & -.133 & .01 & -0.168 & 0.071 & -.122 & .02 & -0.169 & 0.073 & -.117 & .02 & -0.242 & 0.098 & -.122 & .01\end{array}$

\section{Social functioning}

$\begin{array}{lllllllllllllllll}\text { Model } & -0.399 & 0.106 & -.181 & <.001 & -0.351 & 0.091 & -.182 & <.001 & -0.361 & 0.095 & -.180 & <.001 & -0.500 & 0.129 & -.181 & <.001 \\ 1 & & & & & & & & & & & & & & & \\ \begin{array}{l}\text { Model } \\ 2\end{array} & -0.285 & 0.108 & -.130 & .01 & -0.261 & 0.093 & -.135 & .01 & -0.275 & 0.095 & -.137 & .004 & -0.412 & 0.128 & -.149 & .001\end{array}$

\section{Emotional role}

\begin{tabular}{|c|c|c|c|c|c|c|c|c|c|c|c|c|c|c|c|c|}
\hline $\begin{array}{l}\text { Model } \\
1\end{array}$ & 0.040 & 0.121 & .016 & .74 & 0.012 & 0.105 & .005 & .91 & -0.016 & 0.109 & -.007 & .88 & -0.034 & 0.148 & -.011 & .82 \\
\hline $\begin{array}{l}\text { Model } \\
2\end{array}$ & 0.077 & 0.126 & .031 & .54 & 0.039 & 0.109 & .018 & .72 & 0.008 & 0.112 & .004 & .94 & -0.010 & 0.150 & -.003 & .95 \\
\hline
\end{tabular}

\section{Mental health}

$\begin{array}{lllllllllllllllll}\begin{array}{l}\text { Model } \\ 1\end{array} & 0.028 & 0.086 & .016 & .74 & -0.015 & 0.075 & -.010 & .84 & -0.029 & 0.077 & -.018 & .71 & -0.104 & 0.105 & -.047 & .32 \\ \begin{array}{l}\text { Model } \\ 2\end{array} & 0.059 & 0.090 & .034 & .51 & 0.006 & 0.077 & .004 & .94 & -0.010 & 0.079 & -.006 & .90 & -0.087 & 0.106 & -.039 & .41\end{array}$

Physical component

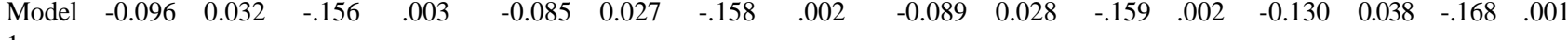




\begin{tabular}{|c|c|c|c|c|c|c|c|c|c|c|c|c|c|c|c|c|}
\hline \multirow{3}{*}{$\begin{array}{l}\text { Dimen- } \\
\text { sions and } \\
\text { models }\end{array}$} & \multicolumn{16}{|c|}{ Percentage of ST accumulated in bouts of different lengths } \\
\hline & \multicolumn{4}{|c|}{$\geq 10$-minute bout } & \multicolumn{4}{|c|}{$\geq 20$-minute bout } & \multicolumn{4}{|c|}{$\geq 30$-minute bout } & \multicolumn{4}{|c|}{$\geq 60$-minute bout } \\
\hline & $\mathrm{B}^{\mathrm{a}}$ & SE & Beta $^{b}$ & $P$ & $\mathrm{~B}$ & SE & Beta & $P$ & B & SE & Beta & $P$ & $\mathrm{~B}$ & SE & Beta & $P$ \\
\hline $\begin{array}{l}\text { Model } \\
2\end{array}$ & -0.083 & 0.033 & -.135 & .01 & -0.075 & 0.028 & -.139 & .01 & -0.079 & 0.029 & -.141 & .01 & -0.119 & 0.039 & -.154 & .002 \\
\hline \multicolumn{17}{|c|}{ Mental component } \\
\hline $\begin{array}{l}\text { Model } \\
1\end{array}$ & -0.023 & 0.050 & -.022 & .64 & -0.031 & 0.043 & -.034 & .47 & -0.039 & 0.045 & -.041 & .39 & -0.065 & 0.061 & -.050 & .28 \\
\hline $\begin{array}{l}\text { Model } \\
2\end{array}$ & 0.003 & 0.052 & .003 & .95 & -0.011 & 0.044 & -.012 & .80 & -0.020 & 0.046 & -.021 & .66 & -0.047 & 0.061 & -.036 & .44 \\
\hline
\end{tabular}

${ }^{\mathrm{a}} \mathrm{B}$ : nonstandardized regression coefficient. Linear regression models were built using Enter method, with SF-36 domains as dependent variables and percentage of ST in different bout lengths as independent variables.

${ }^{b}$ Beta: standardized regression coefficient.

${ }^{\mathrm{c}}$ Model 1: adjusted for age, fat percentage, occupational status, medication for pain, medication for depression, and accelerometer wear time.

${ }^{\mathrm{d}}$ Model 2: analysis controlled for model $1+$ moderate-to-vigorous PA.

Table 4 shows the association of the frequency of bouts of ST of different lengths with the SF-36 domains. A higher frequency of sedentary bouts 20 minutes or longer was associated with worsened vitality and social function (beta=-.12 and -.13, respectively, all $P<.05$ ). A higher frequency of sedentary bouts 30 minutes or longer was associated with worsened physical function, vitality, social function, and PCS scores (beta from -.15 to -.12 , all $P<.05$ ). A higher frequency of sedentary bouts 60 minutes or longer was associated with worsened physical function, physical role, bodily pain, vitality, social function, and PCS scores (beta from -.19 to -.10 , all $P<.05$ ). These associations were independent of MVPA, except for the association with physical role, vitality, and social function in bouts 20 minutes or longer.

Figure 2 shows the combined association of total ST and sedentary bout duration with the SF-36 domains, the PCS, and the MCS. Participants with low total ST and low sedentary bout duration presented better physical function (mean difference 8.73 units, $95 \%$ CI 2.31-15.15), social function (mean difference 10.51 units, 95\% CI 2.59-18.44), and PCS (mean difference 2.71 units, 95\% CI 0.36-5.06) compared to participants with high total ST and high sedentary bout duration (all $P<.02$ ). Additional analyses showed that the differences in the physical function $(P=.045)$ were independent of MVPA. 
Table 4. Association of the frequency of bouts of sedentary time (ST) of different lengths with 36-item Short-Form Health Survey (SF-36) dimensions $(\mathrm{N}=407)$.

\begin{tabular}{|c|c|c|c|c|c|c|c|c|c|c|c|c|c|c|c|c|}
\hline \multirow{3}{*}{$\begin{array}{l}\text { Dimen- } \\
\text { sions and } \\
\text { models }\end{array}$} & \multicolumn{16}{|c|}{ Frequency of bouts (number of bouts/sedentary hours) of different bout lengths } \\
\hline & \multicolumn{4}{|c|}{$\geq 10$-minute bout } & \multicolumn{4}{|c|}{$\geq 20$-minute bout } & \multicolumn{4}{|c|}{$\geq 30$-minute bout } & \multicolumn{4}{|c|}{$\geq 60$-minute bout } \\
\hline & $\mathrm{B}^{\mathrm{a}}$ & SE & Beta $^{b}$ & $P$ & B & SE & Beta & $P$ & B & SE & Beta & $P$ & B & SE & Beta & $P$ \\
\hline \multicolumn{17}{|c|}{ Physical function } \\
\hline $\begin{array}{l}\text { Model } \\
1^{\mathrm{c}}\end{array}$ & 2.386 & 4.237 & .028 & .57 & -10.754 & 5.882 & -.092 & .07 & -18.793 & 7.663 & -.123 & .02 & -61.611 & 16.061 & -.188 & $<.001$ \\
\hline $\begin{array}{l}\text { Model } \\
2^{\mathrm{d}}\end{array}$ & 3.728 & 4.240 & .044 & .38 & -7.970 & 6.002 & -.068 & .19 & -15.416 & 7.820 & -.101 & .049 & -56.713 & 16.208 & -.173 & .001 \\
\hline
\end{tabular}

\section{Physical role}

$\begin{array}{lllllllllllllllll}\text { Model } & 3.602 & 4.575 & .038 & .43 & -4.921 & 6.375 & -.038 & .44 & -12.379 & 8.315 & -.072 & .14 & -35.905 & 17.572 & -.098 & .04 \\ 1 & & & & & & & & & & & & & & & \\ \begin{array}{l}\text { Model } \\ 2\end{array} & 5.161 & 4.572 & .055 & .26 & -1.341 & 6.490 & -.010 & .84 & -8.033 & 8.469 & -.047 & .34 & -29.379 & 17.693 & -.080 & .10\end{array}$

Bodily pain

\begin{tabular}{|c|c|c|c|c|c|c|c|c|c|c|c|c|c|c|c|}
\hline $\begin{array}{l}\text { Model } \\
1\end{array}$ & -1.811 & 3.163 & -.028 & .57 & -6.140 & 4.399 & -.068 & .16 & -9.957 & 5.741 & -.084 & .08 & -31.088 & 12.109 & -.122 \\
\hline $\begin{array}{l}\text { Model } \\
2\end{array}$ & -1.128 & 3.179 & -.017 & .72 & -4.636 & 4.500 & -.051 & .30 & -8.101 & 5.873 & -.068 & .17 & -28.355 & 12.245 & -.111 \\
\hline
\end{tabular}

\section{General health}

$\begin{array}{lllllllllllllllll}\text { Model } & 1.978 & 3.370 & .029 & .56 & -2.700 & 4.696 & -.029 & .57 & -6.395 & 6.132 & -.052 & .30 & -14.374 & 12.987 & -.054 & .27 \\ 1 & & & & & & & & & & & & & & & \\ \begin{array}{l}\text { Model } \\ 2\end{array} & 2.167 & 3.399 & .032 & .52 & -2.433 & 4.818 & -.026 & .61 & -6.189 & 6.289 & -.050 & .33 & -13.956 & 13.166 & -.053 & .29\end{array}$

\section{Vitality}

$\begin{array}{lllllllllllllllll}\text { Model } & -4.226 & 3.928 & -.053 & .28 & -13.300 & 5.441 & -.122 & .02 & -19.025 & 7.101 & -.133 & .01 & -44.261 & 15.015 & -.145 & .003 \\ 1 & & & & & & & & & & & & & & & \\ \begin{array}{l}\text { Model } \\ 2\end{array} & -2.976 & 3.931 & -.038 & .45 & -10.617 & 5.549 & -.097 & .06 & -15.624 & 7.240 & -.109 & .03 & -38.871 & 15.125 & -.127 & .01\end{array}$

\section{Social functioning}

$\begin{array}{lllllllllllllllll}\text { Model } & -2.477 & 5.242 & -.022 & .64 & -19.997 & 7.237 & -.132 & .01 & -30.153 & 9.429 & -.151 & .001 & -73.977 & 19.887 & -.173 & <.001 \\ 1 & & & & & & & & & & & & & & & \\ \begin{array}{l}\text { Model } \\ 2\end{array} & 0.493 & 5.150 & .004 & .92 & -13.339 & 7.267 & -.088 & .07 & -21.717 & 9.472 & -.109 & .02 & -60.962 & 19.730 & -.143 & .002 \\ 2 & & & & & & & & & & & & & \end{array}$

\section{Emotional role}

$\begin{array}{lllllllllllllllll}\begin{array}{l}\text { Model } \\ 1\end{array} & 4.353 & 5.913 & .035 & .46 & 1.973 & 8.246 & .011 & .81 & -2.809 & 10.777 & -.012 & .79 & -4.716 & 22.830 & -.010 & .84 \\ \begin{array}{l}\text { Model } \\ 2\end{array} & 5.130 & 5.958 & .041 & .39 & 3.877 & 8.449 & .023 & .65 & -0.610 & 11.041 & -.003 & .96 & -1.328 & 23.120 & -.003 & .95\end{array}$

\section{Mental health}

\begin{tabular}{|c|c|c|c|c|c|c|c|c|c|c|c|c|c|c|c|c|}
\hline $\begin{array}{l}\text { Model } \\
1\end{array}$ & 5.702 & 4.196 & .065 & .18 & 0.485 & 5.861 & .004 & .93 & -2.013 & 7.659 & -.013 & .79 & -19.328 & 16.197 & -.057 & .23 \\
\hline $\begin{array}{l}\text { Model } \\
2\end{array}$ & 6.372 & 4.225 & .072 & .13 & 1.989 & 6.003 & .016 & .74 & -0.195 & 7.844 & -.001 & .98 & -16.941 & 16.404 & -.050 & .30 \\
\hline \multicolumn{17}{|c|}{ ysical component } \\
\hline $\begin{array}{l}\text { Model } \\
1\end{array}$ & -0.238 & 1.557 & -.008 & .88 & -4.104 & 2.160 & -.097 & .06 & -6.827 & 2.816 & -.122 & .02 & -19.387 & 5.930 & -.162 & .001 \\
\hline
\end{tabular}




\begin{tabular}{|c|c|c|c|c|c|c|c|c|c|c|c|c|c|c|c|c|}
\hline \multirow{3}{*}{$\begin{array}{l}\text { Dimen- } \\
\text { sions and } \\
\text { models }\end{array}$} & \multicolumn{16}{|c|}{ Frequency of bouts (number of bouts/sedentary hours) of different bout lengths } \\
\hline & \multicolumn{4}{|c|}{$\geq 10$-minute bout } & \multicolumn{4}{|c|}{$\geq 20$-minute bout } & \multicolumn{4}{|c|}{$\geq 30$-minute bout } & \multicolumn{4}{|c|}{$\geq 60$-minute bout } \\
\hline & $\mathrm{B}^{\mathrm{a}}$ & SE & Beta $^{b}$ & $P$ & B & SE & Beta & $P$ & B & SE & Beta & $P$ & B & SE & Beta & $P$ \\
\hline $\begin{array}{l}\text { Model } \\
2\end{array}$ & 0.186 & 1.562 & .006 & .91 & -3.222 & 2.208 & -.076 & .15 & -5.754 & 2.877 & -.103 & .046 & -17.763 & 5.989 & -.149 & .003 \\
\hline \multicolumn{17}{|c|}{ Mental component } \\
\hline $\begin{array}{l}\text { Model } \\
1\end{array}$ & 1.309 & 2.431 & .025 & .59 & -1.825 & 3.387 & -.025 & .59 & -4.017 & 4.424 & -.043 & .36 & -11.036 & 9.365 & -.055 & .24 \\
\hline $\begin{array}{l}\text { Model } \\
2\end{array}$ & 1.910 & 2.440 & .037 & .43 & -0.438 & 3.461 & -.006 & .90 & -2.300 & 4.520 & -.024 & .61 & -8.442 & 9.459 & -.042 & .37 \\
\hline
\end{tabular}

${ }^{\mathrm{a}} \mathrm{B}$ : nonstandardized regression coefficient. Linear regression models built using Enter method, with SF-36 domains as dependent variables and percentage of ST in different bout lengths as independent variables.

${ }^{b}$ Beta: standardized regression coefficient.

${ }^{\mathrm{c}}$ Model 1: adjusted for age, fat percentage, occupational status, medication for pain, medication for depression, accelerometer wear time, and ST.

${ }^{\mathrm{d}}$ Model 2: analysis controlled for model $1+$ moderate-to-vigorous PA.

Figure 2. Combined association of total sedentary time (ST) and prolonged sedentary bouts of at least 30 minutes with health-related quality of life. Estimated means represent values after adjustment for age, total body fat percentage, occupational status, medication for pain, medication for depression, and accelerometer-wear time. Common superscripts indicate significant $(P \leq .05)$ differences between groups with the same letter when adjusting for age, body fat percentage, occupational status, medication for pain, medication for depression, and accelerometer-wear time. Asterisks represent significant differences for additional adjustment for moderate-to-vigorous physical activity $(P=.045)$.

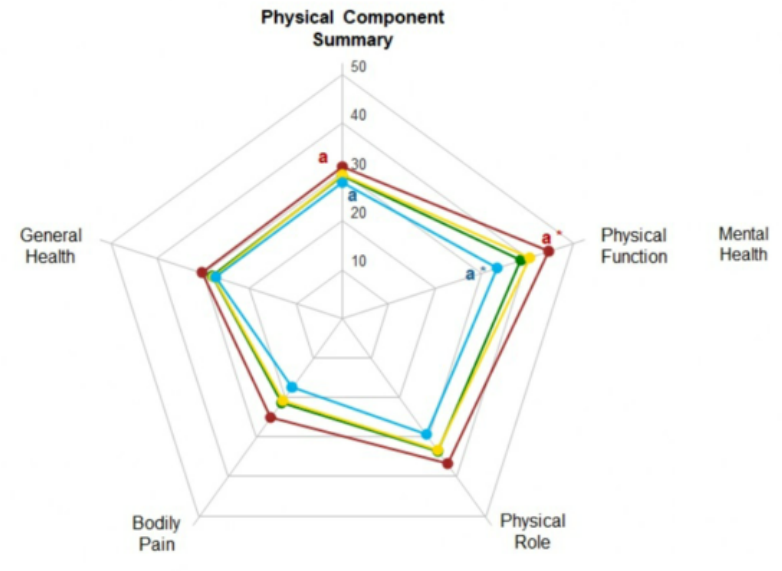

\section{Discussion}

\section{Principal Findings}

The main findings of this study suggest that higher percentages of ST spent in different bout lengths were associated with worsened HRQoL, including physical function, bodily pain, vitality, and social function domains, as well as the PCS. Also, higher frequencies of sedentary bouts were associated with worsened HRQoL, including physical function, bodily pain, vitality, and social function domains, as well as the PCS, especially in longer bout durations. Patients characterized by high total ST and high sedentary bout duration presented worsened physical function, social function, and PCS scores. These associations were generally independent of the MVPA performed for long bout lengths. These findings entail a first step toward the understanding of free-living sedentary behavior

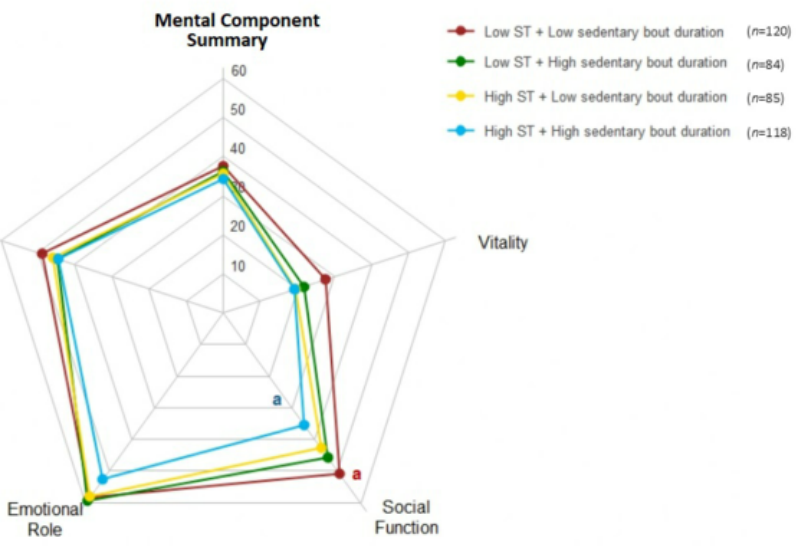

and its association with HRQoL in fibromyalgia. This supports the implementation of mHealth devices, which allow self-monitoring and immediate feedback of daily living behaviors to patients. Future studies might determine whether this approach is successful by reducing prolonged ST in this population.

\section{Limitations}

This study has several limitations that should be underlined. Because our results were derived from a cross-sectional design, the associations cannot be explained via a causal pathway. In addition, due to the large quantity of factors related to HRQoL, it is difficult to ascertain the true nature of the association found between variables. Because only women took part in this study, future studies should investigate whether these associations might extend to men as well. Among its strengths, this study includes a relatively large sample size of women with 
fibromyalgia representative from the south of Spain (ie, Andalusia). According to a recent study, measurement of the actual dose of exercise and daily mobility are essential to establish relationships of these behaviors with health [21]. In this sense, ST and PA were objectively assessed in this study through a wearable tool that enabled researchers to monitor the type, quantity, and quality of everyday activities of patients via accelerometry, which is considered a more reliable technique than questionnaires in the study of fibromyalgia [28]. Future intervention studies with mHealth devices that incorporate in situ information are warranted in this population to ascertain whether fibromyalgia patients change their sedentary behaviors.

\section{Comparison With Prior Work}

To date, most of the previous research on ST and health in fibromyalgia has been limited to the study of total ST [7,29]. In addition, few studies have objectively characterized ST through accelerometry in these patients $[18,30]$ and only one of them [18] reported the values of sustained ST ( $>1$ hour). Ellingson et al demonstrated that sustained ST ( $>60 \mathrm{~min}$ ) was associated with worse pain modulation in fibromyalgia-assessed through magnetic resonance imaging-to a greater extent compared to total ST [18]. Congruently, this study showed negative associations between time spent in sedentary bouts ( $\geq 10 \mathrm{~min}, \geq 20 \mathrm{~min}, \geq 30 \mathrm{~min}$, and $\geq 60 \mathrm{~min}$ ) and the SF-36 body pain dimension. Therefore, we extend the connection between sustained ST and pain to patient-reported instruments. Also, the interruptions of these sedentary bouts might be relevant for pain in this population, given that frequency of sedentary bouts $(\geq 60 \mathrm{~min})$ was negatively associated with bodily pain scores. Following the findings by Ellingson et al, increased pain in sustained ST could be due to the impaired activity in the prefrontal cortices and sensory regions (ie, pre- and postcentral gyri) of these patients [18]. Because the bodily pain domain of the SF-36 not only encompasses objective levels of pain but also the perceived limitations due to it, the contribution of other factors influencing patients' perceptions, such as self-efficacy or pain coping strategies [31], could also take part in this relationship.

Although the influence of patterns of ST has not been explored in fibromyalgia, a direct relationship between increased total ST and fatigue has been described [29]. In agreement with our findings, one previous study in healthy women showed that prolonged ST accumulated in bouts of at least 1 hour were negatively associated with vitality scores of the SF-36 and other fatigue-related variables [32]. Despite the cross-sectional design of these findings that precludes the causal explanation, other experimental studies observed increases in fatigue levels during uninterrupted sitting in adults with overweight and obese status [33] and type 2 diabetes [34], or decreases in fatigue as a result of reducing prolonged sitting [33]. The relationship between ST and fatigue might be explained through physiological, psychological, and social factors that contribute to this multifaceted phenomenon. For instance, prolonged ST could alter the sympathetic nervous system (ie, through a lower heart rate, decreased plasma level of dihydroxyphenylalanine, and increased plasma level of dihydroxyphenylglycol) [33], promote muscle fatigue through sustained activation of low-threshold motor units in sedentary positions [35], or negatively influence sleep quality [7].

In fibromyalgia, there is also a gap in the literature regarding the influence of ST and its patterns on social limitations due to health. For other social-related aspects, Soursa et al stated that patients with fibromyalgia with the lowest PA levels and, presumably, higher levels of ST, had fewer social interactions compared to those doing more PA [36]. No evidence is available on how patterns of ST could influence social function in other populations either, yet interpersonal factors (eg, family, friends, and social networks) are well-known determinants of sedentary behaviors [37]. The passive nature of different sedentary activities (eg, watching television or sitting at the computer) that are accompanied with decreased communication [38] could also lead to poor social networking and participation [39]. Therefore, future research might ascertain whether breaking prolonged ST could positively influence this construct of health (eg, through an increased opportunity to interact with others) or whether strategies aimed at increasing social support may lead to more favorable patterns of accumulation of ST.

To our knowledge, no previous studies have linked patterns of ST to physical function in fibromyalgia. The physical function domain assesses activities of daily living (eg, bathing, dressing, walking several blocks, and lifting or carrying groceries) that typically require a combination of flexibility, strength, and cardiorespiratory fitness, which are related to HRQoL [40]. Previous evidence in adults or older adults showed a decreased physical function, assessed through physical fitness tests, in relation to more deleterious patterns of device-measured ST, such as reduced breaks in ST $[41,42]$, increased sedentary bout duration [41], or increased total prolonged ST [42]. Sedentary periods are linked to skeletal muscle inactivity [43] and are thought to accelerate sarcopenia and loss of aerobic capacity [44], which could negatively affect physical function. Therefore, increases in physical function could be optimized by avoiding the accumulation of ST in prolonged periods and reducing the duration of these ST periods, which needs to be confirmed in future intervention studies.

We observed that, overall, the strength of the associations between the patterns of ST and HRQoL was reduced but still significant when considering MVPA. This finding is congruent with a recent meta-analysis concluding that the deleterious health effects associated with ST generally decrease in magnitude among people with higher levels of PA [13]. Our results also showed that, for certain patterns of ST in shorter bout lengths ( $<60 \mathrm{~min})$, the associations with HRQoL were not significant anymore when considering MVPA. Therefore, performing MVPA could have a protective effect only when ST is accrued in low-duration bouts and could be especially relevant for certain domains of HRQoL, such as bodily pain, physical role, vitality, or social function. Interestingly, meeting the current guidelines of MVPA in bouts of at least 10 minutes was found to neutralize the negative association of prolonged ST with fatigue in healthy women [32]. Hence, it is possible that the patterns of accumulation of MVPA could also influence the capacity of this behavior of counteracting the negative effects of prolonged ST. 


\section{Conclusions}

In conclusion, our findings indicate that higher ST spent in diverse bout lengths and a higher frequency of sedentary bouts, especially in longer bout durations, is associated with worsened HRQoL, more specifically with physical function, bodily pain, vitality, and social function domains, as well as the PCS. Patients that are highly sedentary and present longer sedentary bout durations have worsened physical function, social function, and PCS scores. Although these associations were generally independent of MVPA in long sedentary bout lengths, this intensity of PA could play a positive role when ST is accumulated in shorter bouts. Future intervention studies using mHealth devices that incorporate immediate feedback for users are warranted in this population to ascertain whether fibromyalgia patients change their sedentary behaviors.

\section{Acknowledgments}

We thank the collaborators of the al-Ándalus project and all the members of the Physical Activity for Health Promotion (PAHELP; CTS-1018) research group. We also gratefully acknowledge all the study participants for their collaboration. This study was supported by the Spanish Ministries of Economy and Competitiveness (I+D+i DEP2010-15639; I+D+i DEP2013-40908-R, BES-2014-067612) and the Spanish Ministry of Education (FPU 15/00002). This study was also partially funded by the University of Granada, Plan Propio de Investigación 2016, Excellence actions: Units of Excellence; Unit of Excellence on Exercise and Health (UCEES), by the Junta de Andalucía, Consejería de Conocimiento, Investigación y Universidades and European Regional Development Fund (ERDF), ref SOMM17/6107/UGR, and by the University of Cádiz (Research and Transfer Activity Promotion Program). This study is part of a PhD thesis of the Biomedicine Program of the University of Granada.

\section{Conflicts of Interest}

None declared.

\section{References}

1. Wolfe F, Smythe HA, Yunus MB, Bennett RM, Bombardier C, Goldenberg DL, et al. The American College of Rheumatology 1990 criteria for the classification of fibromyalgia: Report of the Multicenter Criteria Committee. Arthritis Rheum 1990 Feb;33(2):160-172. [doi: 10.1002/art.1780330203] [Medline: 2306288]

2. McLoughlin MJ, Colbert LH, Stegner AJ, Cook DB. Are women with fibromyalgia less physically active than healthy women? Med Sci Sports Exerc 2011 May;43(5):905-912 [FREE Full text] [doi: 10.1249/MSS.0b013e3181fca1ea] [Medline: 20881881]

3. Björnsdóttir SV, Jónsson SH, Valdimarsdóttir UA. Functional limitations and physical symptoms of individuals with chronic pain. Scand J Rheumatol 2013;42(1):59-70. [doi: 10.3109/03009742.2012.697916] [Medline: 23126682]

4. Busch AJ, Webber SC, Brachaniec M, Bidonde J, Bello-Haas VD, Danyliw AD, et al. Exercise therapy for fibromyalgia. Curr Pain Headache Rep 2011 Oct;15(5):358-367 [FREE Full text] [doi: 10.1007/s11916-011-0214-2] [Medline: 21725900]

5. Ellingson LD, Shields MR, Stegner AJ, Cook DB. Physical activity, sustained sedentary behavior, and pain modulation in women with fibromyalgia. J Pain 2012 Feb;13(2):195-206 [FREE Full text] [doi: 10.1016/j.jpain.2011.11.001] [Medline: 22245361]

6. Soriano-Maldonado A, Ruiz JR, Aparicio VA, Estévez-López F, Segura-Jiménez V, Álvarez-Gallardo IC, et al. Association of physical fitness with pain in women with fibromyalgia: The al-Ándalus project. Arthritis Care Res (Hoboken) 2015 Nov;67(11):1561-1570 [FREE Full text] [doi: 10.1002/acr.22610] [Medline: 25939406]

7. Borges-Cosic M, Aparicio V, Estévez-López F, Soriano-Maldonado A, Acosta-Manzano P, Gavilán-Carrera B, et al. Sedentary time, physical activity, and sleep quality in fibromyalgia: The al-Ándalus project. Scand J Med Sci Sports 2019 Feb;29(2):266-274. [doi: 10.1111/sms.13318] [Medline: 30306645]

8. Gavilán-Carrera B, Segura-Jiménez V, Estévez-López F, Álvarez-Gallardo IC, Soriano-Maldonado A, Borges-Cosic M, al-Ándalus Project. Association of objectively measured physical activity and sedentary time with health-related quality of life in women with fibromyalgia: The al-Ándalus project. J Sport Health Sci 2019 May;8(3):258-266 [FREE Full text] [doi: 10.1016/j.jshs.2018.07.001] [Medline: 31193271]

9. Patterson R, McNamara E, Tainio M, de Sá TH, Smith A, Sharp S, et al. Sedentary behaviour and risk of all-cause, cardiovascular and cancer mortality, and incident type 2 diabetes: A systematic review and dose response meta-analysis. Eur J Epidemiol 2018 Sep;33(9):811-829 [FREE Full text] [doi: 10.1007/s10654-018-0380-1] [Medline: 29589226]

10. Owen N, Sparling PB, Healy GN, Dunstan DW, Matthews CE. Sedentary behavior: Emerging evidence for a new health risk. Mayo Clin Proc 2010 Dec;85(12):1138-1141 [FREE Full text] [doi: 10.4065/mcp.2010.0444] [Medline: 21123641]

11. Segura-Jiménez V, Álvarez-Gallardo IC, Estévez-López F, Soriano-Maldonado A, Delgado-Fernández M, Ortega FB, et al. Differences in sedentary time and physical activity between female patients with fibromyalgia and healthy controls: The al-Ándalus project. Arthritis Rheumatol 2015 Nov;67(11):3047-3057 [FREE Full text] [doi: 10.1002/art.39252] [Medline: $\underline{26108350]}$

12. Ekelund U, Steene-Johannessen J, Brown WJ, Fagerland MW, Owen N, Powell KE, et al. Does physical activity attenuate, or even eliminate, the detrimental association of sitting time with mortality? A harmonised meta-analysis of data from more than 1 million men and women. Lancet 2016 Sep;388(10051):1302-1310. [doi: 10.1016/s0140-6736(16)30370-1] 
13. Biswas A, Oh P, Faulkner G, Bajaj R, Silver M, Mitchell M, et al. Sedentary time and its association with risk for disease incidence, mortality, and hospitalization in adults: A systematic review and meta-analysis. Ann Intern Med 2015 Jan 20;162(2):123-132. [doi: 10.7326/M14-1651] [Medline: 25599350]

14. Macfarlane GJ, Kronisch C, Dean LE, Atzeni F, Häuser W, Fluß E, et al. EULAR revised recommendations for the management of fibromyalgia. Ann Rheum Dis 2017 Feb;76(2):318-328. [doi: 10.1136/annrheumdis-2016-209724] [Medline: 27377815]

15. Peddie M, Bone J, Rehrer N, Skeaff C, Gray A, Perry T. Breaking prolonged sitting reduces postprandial glycemia in healthy, normal-weight adults: A randomized crossover trial. Am J Clin Nutr 2013 Aug;98(2):358-366. [doi: 10.3945/ajen.112.051763] [Medline: 23803893]

16. Howard BJ, Fraser SF, Sethi P, Cerin E, Hamilton MT, Owen N, et al. Impact on hemostatic parameters of interrupting sitting with intermittent activity. Med Sci Sports Exerc 2013 Jul;45(7):1285-1291. [doi: 10.1249/MSS.0b013e318285f57e] [Medline: 23439415]

17. Dunstan DW, Kingwell BA, Larsen R, Healy GN, Cerin E, Hamilton MT, et al. Breaking up prolonged sitting reduces postprandial glucose and insulin responses. Diabetes Care 2012 May;35(5):976-983 [FREE Full text] [doi: 10.2337/dc11-1931] [Medline: 22374636]

18. Ellingson LD, Shields MR, Stegner AJ, Cook DB. Physical activity, sustained sedentary behavior, and pain modulation in women with fibromyalgia. J Pain 2012 Feb;13(2):195-206 [FREE Full text] [doi: 10.1016/j.jpain.2011.11.001] [Medline: 22245361]

19. Healy GN, Dunstan DW, Salmon J, Cerin E, Shaw JE, Zimmet PZ, et al. Breaks in sedentary time: Beneficial associations with metabolic risk. Diabetes Care 2008 Apr;31(4):661-666. [doi: 10.2337/dc07-2046] [Medline: 18252901]

20. 2018 Physical Activity Guidelines Advisory Committee. 2018 Physical Activity Guidelines Advisory Committee Scientific Report. Washington, DC: US Department of Health and Human Services; 2018. URL: https://health.gov/paguidelines/ second-edition/report/pdf/PAG Advisory Committee Report.pdf [accessed 2020-02-01]

21. Dobkin B, Dorsch A. The promise of mHealth: Daily activity monitoring and outcome assessments by wearable sensors. Neurorehabil Neural Repair 2011;25(9):788-798 [FREE Full text] [doi: 10.1177/1545968311425908] [Medline: 21989632]

22. Sasaki JE, John D, Freedson PS. Validation and comparison of ActiGraph activity monitors. J Sci Med Sport 2011 Sep;14(5):411-416. [doi: 10.1016/j.jsams.2011.04.003] [Medline: 21616714]

23. Choi L, Ward SC, Schnelle JF, Buchowski MS. Assessment of wear/nonwear time classification algorithms for triaxial accelerometer. Med Sci Sports Exerc 2012 Oct;44(10):2009-2016 [FREE Full text] [doi: 10.1249/MSS.0b013e318258cb36] [Medline: 22525772]

24. Aguilar-Farías N, Brown WJ, Peeters GM. ActiGraph GT3X+ cut-points for identifying sedentary behaviour in older adults in free-living environments. J Sci Med Sport 2014 May;17(3):293-299. [doi: 10.1016/j.jsams.2013.07.002] [Medline: 23932934]

25. Alonso J, Prieto L, Antó JM. The Spanish version of the SF-36 Health Survey (the SF-36 health questionnaire): an instrument for measuring clinical results [Article in Spanish]. Med Clin (Barc) 1995 May 27;104(20):771-776. [Medline: 7783470]

26. Rivera J, Gonzalez T. The Fibromyalgia Impact Questionnaire: A validated Spanish version to assess the health status in women with fibromyalgia. Clin Exp Rheumatol 2004;22(5):554-560. [doi: 10.1007/springerreference_184575]

27. Diaz KM, Goldsmith J, Greenlee H, Strizich G, Qi Q, Mossavar-Rahmani Y, et al. Prolonged, uninterrupted sedentary behavior and glycemic biomarkers among us Hispanic/Latino adults. Circulation 2017 Oct 10;136(15):1362-1373. [doi: 10.1161/circulationaha.116.026858]

28. Segura-Jiménez V, Alvarez-Gallardo IC, Romero-Zurita A, Camiletti-Moirón D, Munguía-Izquierdo D, Carbonell-Baeza A, et al. Comparison of physical activity using questionnaires (leisure time physical activity instrument and physical activity at home and work instrument) and accelerometry in fibromyalgia patients: The Al-Ándalus project. Arch Phys Med Rehabil 2014 Oct;95(10):1903-1911.e2. [doi: 10.1016/j.apmr.2014.05.015] [Medline: 24909586]

29. Segura-Jiménez V, Borges-Cosic M, Soriano-Maldonado A, Estévez-López F, Álvarez-Gallardo IC, Herrador-Colmenero $\mathrm{M}$, et al. Association of sedentary time and physical activity with pain, fatigue, and impact of fibromyalgia: The al-Ándalus study. Scand J Med Sci Sports 2017 Jan;27(1):83-92. [doi: 10.1111/sms.12630] [Medline: 26644186]

30. Bernard P, Hains-Monfette G, Atoui S, Kingsbury C. Differences in daily objective physical activity and sedentary time between women with self-reported fibromyalgia and controls: Results from the Canadian health measures survey. Clin Rheumatol 2018 Aug;37(8):2285-2290. [doi: 10.1007/s10067-018-4139-6] [Medline: 29754181]

31. Lee J, Lee K, Park D, Kim S, Nah S, Lee JH, et al. Determinants of quality of life in patients with fibromyalgia: A structural equation modeling approach. PLoS One 2017;12(2):e0171186 [FREE Full text] [doi: 10.1371/journal.pone.0171186] [Medline: 28158289]

32. Ellingson LD, Kuffel AE, Vack NJ, Cook DB. Active and sedentary behaviors influence feelings of energy and fatigue in women. Med Sci Sports Exerc 2014 Jan;46(1):192-200. [doi: 10.1249/MSS.0b013e3182a036ab] [Medline: 23783259]

33. Wennberg P, Boraxbekk C, Wheeler M, Howard B, Dempsey PC, Lambert G, et al. Acute effects of breaking up prolonged sitting on fatigue and cognition: A pilot study. BMJ Open 2016 Feb 26;6(2):e009630 [FREE Full text] [doi:

10.1136/bmjopen-2015-009630] [Medline: 26920441] 
34. Dempsey PC, Dunstan DW, Larsen RN, Lambert GW, Kingwell BA, Owen N. Prolonged uninterrupted sitting increases fatigue in type 2 diabetes. Diabetes Res Clin Pract 2018 Jan;135:128-133. [doi: 10.1016/j.diabres.2017.11.001] [Medline: 29129482]

35. Hagg G. Static work loads and occupational myalgia: A new explanation model. In: Proceedings of the 8th Congress of the International Society of Electrophysiological Kinesiology. 1991 Presented at: 8th Congress of the International Society of Electrophysiological Kinesiology; August 12-16, 1990; Baltimore, MD p. 141-144.

36. Suorsa K, Lynch-Jordan A, Tran S, Edwards N, Kashikar-Zuck S. Rates of physical activity and perceived social support among young adult women with juvenile-onset fibromyalgia. J Pain 2016 Apr;17(4):S100-S101. [doi: 10.1016/j.jpain.2016.01.311]

37. Chastin SF, De Craemer M, Lien N, Bernaards C, Buck C, Oppert J, DEDIPAC Consortium, Expert Working Group and Consensus Panel. The SOS-framework (Systems of Sedentary behaviours): An international transdisciplinary consensus framework for the study of determinants, research priorities and policy on sedentary behaviour across the life course: A DEDIPAC study. Int J Behav Nutr Phys Act 2016 Jul 15;13:83 [FREE Full text] [doi: 10.1186/s12966-016-0409-3] [Medline: $\underline{27421750]}$

38. Kraut R, Patterson M, Lundmark V, Kiesler S, Mukophadhyay T, Scherlis W. Internet paradox: A social technology that reduces social involvement and psychological well-being? Am Psychol 1998;53(9):1017-1031. [doi:

10.1037//0003-066x.53.9.1017]

39. Kikuchi H, Inoue S, Fukushima N, Takamiya T, Odagiri Y, Ohya Y, et al. Social participation among older adults not engaged in full- or part-time work is associated with more physical activity and less sedentary time. Geriatr Gerontol Int 2017 Nov;17(11):1921-1927. [doi: 10.1111/ggi.12995] [Medline: 28230301]

40. Álvarez-Gallardo IC, Soriano-Maldonado A, Segura-Jiménez V, Estévez-López F, Camiletti-Moirón D, Aparicio V, et al. High levels of physical fitness are associated with better health-related quality of life in women with fibromyalgia: The al-Ándalus project. Phys Ther 2019 Nov 25;99(11):1481-1494. [doi: 10.1093/ptj/pzz113] [Medline: $\underline{31392995]}$

41. van der Velde JH, Savelberg HH, van der Berg JD, Sep SJ, van der Kallen CJ, Dagnelie PC, et al. Sedentary behavior is only marginally associated with physical function in adults aged 40-75 years: The Maastricht study. Front Physiol 2017;8:242 [FREE Full text] [doi: 10.3389/fphys.2017.00242] [Medline: $\underline{28487660}$ ]

42. Dogra S, Clarke JM, Copeland JL. Prolonged sedentary time and physical fitness among Canadian men and women aged 60 to 69. Health Rep 2017 Feb 15;28(2):3-9 [FREE Full text] [Medline: 28198983]

43. Bergouignan A, Latouche C, Heywood S, Grace M, Reddy-Luthmoodoo M, Natoli A, et al. Frequent interruptions of sedentary time modulates contraction- and insulin-stimulated glucose uptake pathways in muscle: Ancillary analysis from randomized clinical trials. Sci Rep 2016 Aug 24;6:32044 [FREE Full text] [doi: 10.1038/srep32044] [Medline: 27554943]

44. Thyfault JP, Du M, Kraus WE, Levine JA, Booth FW. Physiology of sedentary behavior and its relationship to health outcomes. Med Sci Sports Exerc 2015;47(6):1301-1305. [doi: 10.1249/mss.0000000000000518]

\author{
Abbreviations \\ cpm: counts per minute \\ ERDF: European Regional Development Fund \\ FIQR: Revised Fibromyalgia Impact Questionnaire \\ HRQoL: health-related quality of life \\ MCS: mental component summary \\ MVPA: moderate-to-vigorous physical activity \\ PA: physical activity \\ PAHELP: Physical Activity for Health Promotion \\ PCS: physical component summary \\ SF-36: 36 -item Short-Form Health Survey \\ ST: sedentary time \\ UCEES: Unit of Excellence on Exercise and Health
}

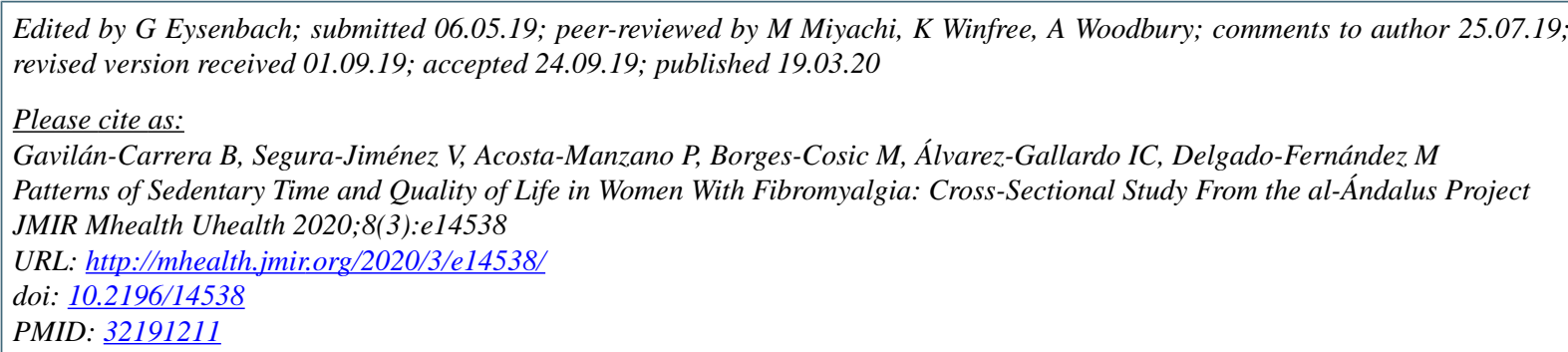


CBlanca Gavilán-Carrera, Víctor Segura-Jiménez, Pedro Acosta-Manzano, Milkana Borges-Cosic, Inmaculada C Álvarez-Gallardo, Manuel Delgado-Fernández. Originally published in JMIR mHealth and uHealth (http://mhealth.jmir.org), 19.03.2020. This is an open-access article distributed under the terms of the Creative Commons Attribution License (https://creativecommons.org/licenses/by/4.0/), which permits unrestricted use, distribution, and reproduction in any medium, provided the original work, first published in JMIR mHealth and uHealth, is properly cited. The complete bibliographic information, a link to the original publication on http://mhealth.jmir.org/, as well as this copyright and license information must be included. 